Received: 19.05 .2019

Revised: 28.06 .2019

Accepted: 28.06 .2019

DOI: $10.17804 / 2410-9908.2019 .3 .025-040$

\title{
A SUFFICIENT CONDITION FOR THE ABSENCE OF STRONG AND WEAK DISCONTINUITIES IN GAS FLOWS IN FLAT CHANNELS
}

\author{
E. Yu. Prosviryakov \\ Institute of Engineering Science, Ural Branch of the Russian Academy of Sciences, \\ 34 Komsomolskaya St., Ekaterinburg, 620049, Russian Federation \\ iD https://orcid.org/0000-0002-2349-7801 《evgen_pros@mail.ru \\ Corresponding author. E-mail: evgen_pros@mail.ru \\ Address for correspondence: 34 Komsomolskaya St., Ekaterinburg, 620049, Russian Federation
} Tel.: +7 (343) 37420 38; fax: 3745330

The paper brings together all the assumptions about the properties of discontinuous flows of an ideal (perfect) gas, both formulated in textbooks and not formulated in the available literature, but having actually been long and effectively used. In addition, some new assumptions are physically grounded and formulated for the plane steady-state flow. All these properties are formulated in the form of a continuous continuum hypothesis for plane stationary flows of an ideal (perfect) gas. The hypothesis is formulated in such a way that, to justify the calculations and reasoning in the solution of problems, it would be possible not to resort to physical considerations every time again, but to rely on the "ready" statements of the hypothesis. Using the statements of this hypothesis, a sufficient condition for the impossibility of the existence of discontinuities in the flows occurring in flat channels is obtained. In the derivation of sufficient conditions, were use only the statements of the hypothesis, without involving any additional physical considerations.

Keywords: continuous continuum, perfect gas, discontinuous gas flows, smoothness of flow parameters.

\section{Acknowledgment}

We are grateful to Prof. A. L. Stasenko (TsAGI) and Dr. G. B. Sizykh (MIPT) for the discussion of the "hypothesis" and useful remarks.

\section{References}

1. $\quad$ Loitsyanskii L.G. Mechanics of Liquids and Gases. Pergamon Press, 1966.

2. Bers L. Mathematical Aspects of Subsonic and Transonic Gas Dynamics. John Wiley \& Sons, Inc., New York, Chapman \& Hall, Ltd, London, 1958.

3. Kochin N.K., Kibel I.A., Roze N.V. Theoretical Hydromechanics. Wiley Interscience, 1964.

4. Batchelor G.K. An Introduction to Fluid Dynamics. University Press, Cambridge, 1970. DOI: $10.1017 / \mathrm{CBO} 9780511800955$.

5. Sedov L.I. Mechanics of Continuous Media. World Sci., River Edge, NJ, 1997.

6. Nikol'skii A.A., Taganov G.I. The motion of a gas in a local supersonic zone and some conditions for the breakdown of potential flow. Prikl. Mat. Mekh., 1946, vol. 10, no. 4.

7. Hopf E. Elementare Bemerkungen über die Lösungen partieller Differentialgleichungen zweiter Ordnung vom Elliptischen Typus. Sitzungsberichte der Preussischen Akademie der Wissenschaften, 1927, vol. 19, pp. 147-152.

8. Miranda C. Partial Differential Equations of Elliptic Type. Springer-Verlag Berlin Heidelberg, 1970. 
9. Gilbarg D., Shiffman M. On bodies achieving extreme values of the critical Mach number I. J. Rat. Mech. and Analysis, 1954, vol. 3, iss. 2, pp. 209-230.

10. Kraiko A.N. Planar and axially symmetric configurations which are circumvented with the maximum critical mach number. Journal of Applied Mathematics and Mechanics, 1987, vol. 51, iss. 6, pp. 723-730. DOI: 10.1016/0021-8928(87)90131-6.

11. Rosenhead L. The formation of vortices from a surface of discontinuity. P. Roy. Soc. Lond., 1931, A134, pp. 170-192.

12. Belotserkovsky S.M., Nisht M.I. Otryvnoe i bezotryvnoe obtekanie tonkikh krylyev idealnoy zhidkostyu [Separated and Separationless Ideal-Fluid Flows past Thin Wings]. Moscow, Nauka Publ., 1978, 352 p. (In Russian).

13. Cottet G.-H., Koumoutsakos P. Vortex Methods: Theory and Practice. Cambridge University Press, 2000.

14. Gutnikov V.A., Lifanov I.K., Setukha A.V. Simulation of the aerodynamics of buildings and structures by means of the closed vortex loop method. Fluid Dynamics, 2006, vol. 41, no. 4, pp. 555-567. DOI: 10.1007/s10697-006-0073-4.

15. Fihtengolts G.M. Kurs differentsialnogo i integralnogo ischisleniya [A Course in Differential and Integral Calculus, vol. 2]. Moscow, Fizmatlit Publ., 2001. (In Russian).

16. Munk M., Prim R. On the multiplicity of steady gas flows having the same streamline pattern. Proc. Nat. Acad. Sci. USA, 1947, vol. 33, pp. 137-141.

17. Sizykh G.B. The Criterion of the Presence of a Stagnation Point in a Plane Irrotational Flow of Inviscid Gas. Trudy MFTI, 2015, vol. 7 (2), 108-112. (In Russian).

18. Golubkin V.N., Sizykh G.B. Property of the extreme pressure values in plane subsonic flows. Trudy MFTI, 2016, vol. 8, no. 4, pp. 149-154. (In Russian). 
Подана в журнал: 19.05.2019

УДК 533.6.011

DOI: $10.17804 / 2410-9908.2019 .3 .025-040$

\title{
ДОСТАТОЧНОЕ УСЛОВИЕ ОТСУТСТВИЯ СИЛЬНЫХ И СЛАБЫХ РАЗРЫВОВ ПРИ ТЕЧЕНИИ ГАЗА В ПЛОСКИХ КАНАЛАХ
}

\author{
Е. Ю. Просвиряков \\ Институт машиноведения Уральского отделения Российской академии наук, \\ ул. Комсомольская, 34, г. Екатеринбург, Российская Федерация \\ (iD https://orcid.org/0000-0002-2349-7801 @ evgen_pros@mail.ru \\ Ответственный автор. Электронная почта: evgen_pros@mail.ru \\ Адрес для переписки: ул. Комсомольская, 34, Екатеринбург, Российская Федерация \\ Тел.: +7 (343) 374-20-38; факс: 374-53-30
}

\begin{abstract}
В работе собраны все предположения о свойствах разрывных течений идеального совершенного газа: сформулированные в учебниках и не сформулированные в доступной литературе, но фактически уже давно эффективно использующиеся. Кроме того, для плоского стационарного течения физически обоснованы и сформулированы некоторые новые предположения. Все эти свойства представлены в виде гипотезы непрерывной сплошной среды для плоских стационарных течений идеального совершенного газа. Гипотеза сформулирована таким образом, чтобы для обоснования выкладок и рассуждений при решении задач можно было не прибегать всякий раз заново к физическим соображениям, а опираться на готовые положения гипотезы. С использованием положений гипотезы получено достаточное условие невозможности существования разрывов в течениях, происходящих в плоских каналах. При выводе этого достаточного условия были использованы только положения гипотезы и не привлекались никакие дополнительные физические соображения.
\end{abstract}

Ключевые слова: непрерывная сплошная среда, идеальный совершенный газ, разрывные течения газа, гладкость параметров течения.

\section{1. Введение}

Ряд практически важных задач газовой динамики не имеет решений в классе гладких (непрерывно дифференцируемых) функций. Плотность и скорость могут иметь разрывы на поверхности, разделяющей две сонаправленные газовые струи с одинаковым давлением. В сверхзвуковых течениях могут существовать поверхности слабых разрывов (разрывы производных без разрыва параметров) и скачки уплотнения - поверхности, после пересечения которых течение становится дозвуковым. Представление о поверхностях вводится, чтобы не рассматривать зоны течений, являющиеся тонкими слоями порядка средней длины свободного пробега молекул с большими градиентами гидродинамических параметров. Дело в том, что для идеального (роль диффузии молекул пренебрежимо мала, вязкость и теплопроводность считаются равными нулю) уравнения Эйлера учитывают только конвективный перенос и поэтому не позволяют исследовать структуру узких переходных зон в рамках гладких функций. Представление о поверхностях оправдывается тем, что во многих задачах толщина этих слоев пренебрежимо мала по сравнению с характерными размерами области течения, и ее можно считать равной нулю, рассматривая упомянутые слои как поверхности разрыва. Чтобы учесть возможность присутствия в решениях поверхностей разрыва, решения ищутся в наиболее простом для применения математического анализа классе негладких функций в классе кусочно-гладких функций. Во всех точках течения, в том числе и на поверхностях разрывов, должны соблюдаться законы сохранения массы, импульса и энергии. В зонах 
гладкости для идеального газа эти законы равносильны уравнениям Эйлера, уравнению неразрывности и уравнению состояния для движущихся макрочастиц газа. Считается, что разрывы могут располагаться только на конечном количестве кусочно-гладких поверхностей. И хотя этот класс не исчерпывает всех возможных с точки зрения математики расположений точек разрыва, дальнейшего ослабления гладкости до сих пор не требовалось. Именно такие решения рассматриваются в ставших классическими учебниках и монографиях [1-4]. Причина этого так объяснена Л. И. Седовым: «Снятие требования непрерывности и допущение о кусочной гладкости искомых решений обеспечивает при соответствующей постановке задачи существование и единственность решения. Получающиеся разрывные решения могут хорошо соответствовать реальным эффектам, наблюдаемым на практике» $[5$, гл. VII]. Таким образом, рассмотрение течений в классе кусочно-гладких функций соответствует широкому классу практически важных задач газовой динамики.

Исследование общих свойств течений газа в классе кусочно-гладких функций предполагает возможность наличия разрывов давления, плотности и скорости $(p, \rho, \mathbf{V})$ или разрывов их производных во внутренних точках течения. В постановках соответствующих краевых задач ни количество разрывов, ни места их расположения заранее не указаны и должны быть определены в процессе решения. В этой связи следует упомянуть доказанную в настоящее время гипотезу Буземанна - Гудерлея - Франкля о том, что гладкое околозвуковое течение вокруг произвольного профиля невозможно [2, гл. V]; в таком течении обязательно будет поверхность разрыва.

При исследовании течений жидкости и газа нередко прибегают к использованию физических соображений. С одной стороны, результат, полученный с использованием физических соображений, зачастую значительно превосходит результаты не только математиков, опирающихся на известные закономерности, но превосходит и результаты очень опытных физиков, которые понимают сложность задачи и не осмеливаются утверждать, что им априори понятна физическая картина явления. Но если интуиция подведет, то такой результат может оказаться ошибочным. Поэтому использование физических соображений, относящихся, как правило, к специфичной именно для рассматриваемой задачи ситуации, впервые сформулированных по ходу исследования и не прошедших широкого обсуждения, представляется недопустимым. При этом некоторые физические соображения, не содержащиеся в сформулированных известных законах, давно подтверждены правильностью выводов, которые получены с их использованием, и требуют лишь формализации. На такие физические соображения вполне допустимо опираться при исследованиях. Речь идет о физических допущениях, проверенных практикой и апробированных известными учеными.

В статье предпринята попытка собрать воедино все предположения о свойствах разрывных плоских стационарных течений газа: и сформулированные в учебниках [1-5], и не сформулированные в доступной литературе, но фактически уже давно эффективно использующиеся, предположения. Кроме того, для плоского стационарного течения физически обоснованы и сформулированы некоторые новые предположения (они касаются вопросов гладкости параметров течения и линий разрывов). Все эти свойства (известные и предложенные в статье) сформулированы ниже в виде гипотезы непрерывной сплошной среды для плоских стационарных течений идеального совершенного газа. Подчеркнем, что речь идет именно о предположениях (о моделях), а не о различных теоремах газовой динамики, которые доказаны, например, на основе уравнений Эйлера и предположения о сохранении энтропии на линиях тока (в зонах гладких параметров течения). Речь идет о самих уравнениях Эйлера и о различных физически обоснованных свойствах, на основе которых доказываются подобные теоремы. Поэтому среди положений гипотезы отсутствуют, например, теоремы Гельмгольца, Никольского - Таганова [6], дозвуковой принцип максимума скорости и другие известные утверждения, которые являются следствиями уравнений Эйлера и физически обоснованных утверждений. Гипотеза сформулирована таким образом, чтобы 
для обоснованности выкладок и рассуждений при решении задач и при теоретических исследованиях можно было опираться на положения гипотезы, а не манипулировать физическими соображениями по ходу исследования. Это позволит привлечь к исследованиям математиков, что является одной из главных целей данной статьи. Гипотеза представлена для обсуждения, и, возможно, некоторые ее положения будут изменены в будущем. При этом, как представляется, причиной для внесения изменений в гипотезу будет не сомнительность того или иного положения, а избыточность или недостаточность положений гипотезы для математического (без привлечения дополнительных физических соображений) исследования плоских течений газа.

В качестве примера состоятельности гипотезы с ее использованием были рассмотрены краевые задачи, в которых решения ищутся в классе кусочно-гладких функций (то есть допускается наличие разрывов), но, в силу положений гипотезы, существование разрывов становится невозможным при надлежащем выборе граничных условий. Интерес к таким течениям вызван тем, что уравнения Эйлера выполняются во всех точках, и известные математические теоремы из теории уравнений в частных производных (например принципы максимума) позволяют делать интересные выводы об общих свойствах таких течений. В результате использования положений гипотезы (без привлечения дополнительных физических соображений) получено достаточное условие невозможности существования разрывов в течениях, происходящих в плоских каналах.

\section{2. Гипотеза непрерывной сплошной среды для плоских стационарных течений идеального совершенного газа}

Прежде чем сформулировать гипотезу, напомним о некоторых положениях, которые уже давно де-факто являются общепризнанными. Свойство кусочной гладкости гидродинамических параметров постоянно упоминается и используется во всей классической литературе. Условия сохранения массы, импульса и энергии при протекании вещества через поверхность разрыва или равенство давлений на разных сторонах тангенциального разрыва четко указаны в [1-5]. Иначе обстоит дело с непрерывностью вторых производных. Речь идет о предположении, которое де-факто используется, но обычно не оговаривается. Оно состоит в том, что в области гладкости (области непрерывности первых производных) вторые производные также считаются непрерывными. Однако есть одно отличие. Если кусочная гладкость предполагает возможность непрерывного продолжения производных из зоны гладкости на соответствующую сторону поверхности разрыва, то непрерывность вторых производных гидродинамических параметров касается только гладких участков поверхностей разрыва. Предположение о непрерывности вторых производных внутри областей гладкости подтверждается тем, что оно позволяет объяснить некоторые экспериментально наблюдаемые явления, а также тем, что оно эффективно используется в численных методах. Приведем четыре примера.

Первый пример. В [2, гл. II] объяснено, почему компоненты скорости безвихревого дозвукового стационарного течения ( $M<1, M$ - число Маха) сжимаемой жидкости принимают свои наибольшие значения на границе области течения (дозвуковой принцип максимума модуля скорости). Объяснение основано на теореме Хопфа $[7,8]$ и поэтому предполагает непрерывность вторых производных компонент скорости.

Второй пример. В статье [9] получено необходимое условие существования внутренних звуковых точек для плоских и осесимметричных течений, в которых $M \leq 1$. Оно объясняет тот факт, что если в потоке $M \leq 1$ существует внутренняя звуковая точка, то эта точка лежит на прямой звуковой линии, состоящей из звуковых точек. Эта прямая звуковая линия в каждой точке перпендикулярна скорости газа и не может заканчиваться внутри потока. В [10] это свойство названо «свойством прямолинейности» и было использовано для построения плоских и осесимметричных конфигураций с максимально возможным критическим 
числом Маха $M^{*}$. Доказательство свойства прямолинейности существенным образом опирается на предположение о непрерывности вторых производных компонент скорости, хотя специально в [9] не оговаривается.

Третий пример. Как в стационарном, так и в нестационарном безвихревом течении несжимаемой жидкости давление не может достигать своего минимума во внутренних точках течения. Это показано, например в [4, гл. 6], чтобы объяснить, почему кавитация зарождается именно на поверхности обтекаемых тел. Для соответствующего доказательства недостаточно гладкости или существования вторых производных. Достаточным условием для обоснованности доказательства является непрерывность вторых производных давления.

И наконец, четвертый самый известный пример. Теоремы Гельмгольца о вихрях лежат в основе известного и проверенного на практике численного метода дискретных вихрей [11-14]. При доказательстве теорем Гельмгольца используется факт равенства нулю ротора от градиента давления. И снова достаточным условием для обоснованности доказательства является непрерывность вторых производных давления.

С математической точки зрения из кусочной гладкости не следует существование и тем более кусочная непрерывность вторых производных. Например, первообразная функции Ван-дер-Вардена [15, гл. ХII] непрерывно дифференцируема, и при этом ни в одной точке она не имеет второй производной. Поэтому утверждение о существовании и непрерывности вторых производных в областях, где существуют и непрерывны первые производные, следует считать еще одной общепризнанной гипотезой, наряду с предположением о кусочной гладкости.

Рассмотрим плоское стационарное дозвуковое течение без точек торможения. В таком течении не может быть точек, в которых давление и плотность равны нулю. Действительно, в стационарных течениях могут существовать два типа зон. В зонах первого типа частицы газа (не молекулы, а макрочастицы) находятся конечное время. Соответствующие этим частицам линии тока начинаются и заканчиваются на границах течения. Для таких частиц пренебрежение диффузией молекул оправдывается конечным временем процесса. В зонах второго типа частицы газа, вообще говоря, движутся, но все время остаются в области течения. В связи с неограниченным временем процесса (для стационарных течений) диффузией молекул в таких зонах пренебрегать нельзя. Для дозвуковых течений, по сравнению со сверхзвуковыми течениями, этот эффект выражен сильней, поскольку в дозвуковом потоке скорость частиц газа ниже средней скорости молекул. По этой же причине нельзя пренебречь диффузией молекул из соседних зон в зону вакуума, которая могла появляться, пока течение устанавливалось. Вакуум не может соседствовать с дозвуковой зоной ненулевой плотности в стационарных дозвуковых течениях. Поэтому среди возможных решений уравнений Эйлера реальности могут соответствовать только те решения, в которых давление и плотность не обращаются в ноль, по крайней мере, во внутренних точках подобластей с гладкими параметрами $(p, \rho, \mathbf{V})$.

Из лагранжева представления о движении частицы газа следует, что если скорость в точке не равна нулю, то эта точка не может представлять собой одну изолированную точку разрыва, окруженную областью гладких параметров.

Поскольку в данной статье речь идет о плоских течениях, будем говорить не о поверхностях разрыва, а об их проекциях на плоскость течения - о линиях разрыва. Предположение о кусочной гладкости линий разрывов, само по себе, значительно сужает класс вариантов расположения точек разрыва. Однако исходя из физических соображений, можно еще более упростить вид рассматриваемых кривых. Как уже было замечено, разрыв, строго говоря, является не линией, а «размытой» полосой, шириной не менее длины свободного пробега. Площадь полосы не может быть бесконечной, если полоса расположена в ограниченной области течения. Поэтому линию разрыва в ограниченной области можно считать имеющей ограниченную длину. Кроме того, поскольку кривизна полосы ограничена, можно считать, 
что если кусочно-гладкая кривая разрыва не замкнута сама на себя, то у нее есть начало и конец с определенными предельными положениями касательных векторов.

Сказанное выше, а также широко известные из учебников положения, обосновывают содержательность рассмотрения плоского стационарного течения идеального совершенного газа при отсутствии внешних сил в следующей постановке, допускающей существование линий разрыва. Ниже используются обозначения: $\mathbf{V}$ - скорость; $\rho>0$ - плотность; $k>0-$ показатель адиабаты; $p$ - давление.

\section{3. Гипотеза непрерывной сплошной среды для плоских стационарных течений идеального совершенного газа}

Пусть $O x y$ - произвольная прямоугольная декартова система координат, лежащая в плоскости течения. Ограниченная область течения газа может состоять из объединения не более чем конечного числа подобластей, в каждой из которых гидродинамические параметры $p, \rho, \mathbf{V}$ являются дважды непрерывно дифференцируемыми функциями координат $x$ и $y$. Границы всей области течения и границы, разделяющие подобласти, являются кусочногладкими линиями. Если область течения ограничена, то все эти линии имеют конечную длину, а кривизна гладких участков ограничена сверху. Параметры $p, \rho, \mathbf{V}$ допускают непрерывное продолжение из внутренних точек на «свою» сторону границы подобласти. На гладкие участки линии разрыва также возможны непрерывные продолжения производных $p, \rho, \mathbf{V}$. Эти непрерывные продолжения на разных сторонах границы, разделяющей подобласти, вообще говоря, могут не совпадать. Границы, разделяющие подобласти, являются линиями разрыва хотя бы одного из гидродинамических параметров или его первых производных. Если в некоторой области все три параметра $p, \rho, \mathbf{V}$ являются гладкими, т. е. непрерывны вместе со своими первыми производными по координатам $x$ и $y$, то в этой области существуют и непрерывны вторые производные $p, \rho, \mathbf{V}$. Плотность и давление во внутренних точках подобластей гладкости не обращаются в ноль. Упомянутые подобласти могут содержаться одна внутри другой или содержать во внутренних точках незамкнутую линию разрыва, не являющуюся границей какой-либо подобласти. Если в течении нет точек торможения, то в упомянутых областях гладкости не может находиться изолированная точка разрыва. Предположение о возможности непрерывного продолжения производных не относится к точкам излома кусочно-гладких линий разрыва. Если кусочно-гладкая кривая разрыва не замкнута сама на себя, то у нее есть начало и конец с определенными предельными положениями касательных векторов.

На любом гладком участке линии разрыва, который пересекается макрочастицами газа, должны быть непрерывны следующие четыре функции, зависящие от параметров $p, \rho, \mathbf{V}$ :

$$
V_{\tau} ; \quad \rho V_{n} ; \quad p+\rho V_{n}^{2} ; \quad\left(\frac{k}{k-1} p+\rho \frac{V_{n}^{2}}{2}+\rho \frac{V_{\tau}^{2}}{2}\right) V_{n},
$$

где $V_{\tau}$ и $V_{n} \neq 0$ - касательная и нормальная к линии разрыва составляющие вектора скорости $\mathbf{V}$. Если величина $p \cdot \rho^{-k}$ для макрочастицы газа при переходе линии разрыва меняется скачкообразно, то только в сторону увеличения.

Если через гладкий участок линии разрыва не происходит перенос макрочастиц газа $\left(V_{n}=0\right)$, то единственным условием на таком участке является непрерывность давления $p$.

Движение газа внутри каждой подобласти с гладкими параметрами описывается уравнениями Эйлера, записанными, например, в форме Громеки - Ламба [1]: 


$$
[\operatorname{rot} \mathbf{V} \times \mathbf{V}]=-\frac{\nabla p}{\rho}-\nabla\left(\frac{V^{2}}{2}\right) ; \quad V=|\mathbf{V}|
$$

уравнением неразрывности [1]

$$
\operatorname{div}(\rho \mathbf{V})=0
$$

и уравнением адиабатического движения частиц газа

$$
\mathbf{V} \cdot \nabla\left(p \cdot \rho^{-k}\right)=0
$$

Примером ограниченной (плоской) области, упоминаемой в приведенной формулировке, может быть область течения в плоском канале, ограниченная входным и выходным сечениями, а также стенками канала.

Ниже из этой гипотезы будут получены интересные выводы о линиях разрыва в плоских дозвуковых течениях без точек торможения.

\section{4. Плоские дозвуковые течения без точек торможения}

В следующих разделах будут исследованы линии разрыва, расположенные во внутренних точках течения, в котором скорость газа всюду ограничена снизу некоторой положительной константой $\delta_{V}$ и не достигает скорости звука:

$$
\begin{gathered}
V \geq \delta_{V}>0 \\
k p-\rho V^{2}>0 .
\end{gathered}
$$

Эти два неравенства являются формальным определением термина «дозвуковое течение без точек торможения».

\section{5. Тангенциальные разрывы}

Независимо от того, какой гидродинамический параметр или его производная терпит разрыв, все возможные ситуации разделяются на два типа: есть разрыв плотности или нет.

Если на гладком участке линии разрыва скачкообразно меняется плотность и скорость имеет ненулевую нормальную к этой линии составляющую, то такой разрыв называют скачком уплотнения [1-5]. С одной из его сторон скорость будет выше скорости звука, что противоречит условию (5), и такой разрыв внутри дозвукового течения невозможен. Если же скорость не имеет нормальной к линии разрыва составляющей, то можно привести пример течения с разрывом плотности при соблюдении условий (4) и (5). Это упомянутое во введении дозвуковое течение двух параллельных, имеющих общую поверхность (для плоских течений - общую линию) соприкосновения, однородных потоков газа разной плотности, но одинакового давления. На таких линиях разрыва, в которых скорость не имеет нормальной к линии разрыва составляющей, не исключена возможность разрыва скорости. Но предельные значения вектора скорости с разных сторон линии разрыва, согласно (4), не равны нулю и определяют предельные положения линий тока. Следовательно, если в течении есть линия разрыва плотности и выполнены условия (4) и (5), то на гладком участке этой линии лежат предельные положения линий тока (тангенциальный разрыв). Заметим, что это верно для 
разных вариантов разрывности или непрерывности остальных гидродинамических параметров на линии разрыва плотности.

Теперь рассмотрим гладкий участок линии разрыва, на котором плотность непрерывна. При этом никаких предположений о непрерывности $p, \mathbf{V}$ или о непрерывности производных $p, \rho, \mathbf{V}$ делать не будем. Возможны два случая в зависимости от того, равна ли нулю нормальная к линии разрыва составляющая скорости.

Первый случай. Скорость имеет ненулевую нормальную к линии разрыва составляющую. Тогда частицы газа переносят через разрыв массу, импульс и полную энтальпию. Повторяя известные [1-5] выкладки для получения условий на прямых и косых скачках уплотнения, получим, что из непрерывности плотности следует непрерывность давления и вектора скорости. Покажем, что при этом производные гидродинамических параметров также будут непрерывны.

Расположим начало прямоугольной декартовой системы координат $O x y$ в произвольной внутренней точке гладкого участка рассматриваемой линии разрыва. Ось $O x$ направим по касательной к этой линии. В силу непрерывности гидродинамических параметров на линии разрыва продолжения их производных по координате $x$ на обе стороны этой линии будут одинаковы в начале координат. Уравнения (1) - (3) также выполнены с обеих сторон от линии разрыва. Если записать их в системе координат $O x y$, учитывая непрерывность $p, \rho, \mathbf{V}$ и равенство производных $p, \rho, \mathbf{V}$ по координате $x$ в начале координат, то можно заключить, что в начале координат при переходе через линию разрыва остаются неизменными следующие четыре величины (здесь $V_{x}, V_{y}$ - компоненты скорости $\mathbf{V}$ ):

$$
q_{1}=V_{y} \frac{\partial}{\partial y} V_{x} ; q_{2}=V_{y} \frac{\partial}{\partial y} V_{y}+\frac{1}{\rho} \frac{\partial}{\partial y} p ; q_{3}=V_{y} \frac{\partial}{\partial y} \rho+\rho \frac{\partial}{\partial y} V_{y} ; q_{4}=\rho V_{y} \frac{\partial}{\partial y} p-k p V_{y} \frac{\partial}{\partial y} \rho
$$

Эти равенства задают систему линейных уравнений относительно производных $\frac{\partial}{\partial y} V_{x}$; $\frac{\partial}{\partial y} V_{y} ; \frac{\partial}{\partial y} p ; \frac{\partial}{\partial y} \rho$. Определитель системы равен $V_{y}^{2}\left(k p-\rho V_{y}^{2}\right)$. Предположительно скорость имеет ненулевую нормальную к поверхности разрыва составляющую, т. е. $V_{y} \neq 0$. Кроме того, $V_{y}^{2} \leq V^{2}$, что вместе с (5) обеспечивает положительность рассматриваемого определителя. Следовательно, производные $\frac{\partial}{\partial y} V_{x} ; \frac{\partial}{\partial y} V_{y} ; \frac{\partial}{\partial y} p ; \frac{\partial}{\partial y} \rho$ однозначно определяются через $q_{1}$; $q_{2} ; q_{3}$ и $q_{4}$, а это означает их непрерывность в начале координат. Расположение начала координат было выбрано произвольно, поэтому пространственные производные непрерывны во всех внутренних точках гладкого участка линии. То есть на рассматриваемой линии непрерывны как параметры течения $p, \rho, \mathbf{V}$, так и их производные. Согласно принятому в конце предыдущего раздела предположению о свойствах разрывных решений, это означает, что на этой линии непрерывны и вторые производные $p, \rho, \mathbf{V}$. Итак, в рассматриваемых течениях не может быть линий разрывов, на которых плотность непрерывна, а скорость имеет ненулевую нормальную к линии разрыва составляющую.

Второй случай. Плотность непрерывна, а скорость имеет нулевую нормальную к линии разрыва составляющую. В этом случае давление тоже непрерывно [5]. Используем обозначения только что проведенного доказательства. Тогда $V_{y}=0$, и определитель 
$V_{y}^{2}\left(k p-\rho V_{y}^{2}\right)$ системы равен нулю. Поэтому из системы не следует непрерывность производных $\frac{\partial}{\partial y} V_{x}$ и $\frac{\partial}{\partial y} \rho$. Более того, несложно привести пример стационарного течения со слабым тангенциальным разрывом. Для этого достаточно рассмотреть любое течение с гладкими параметрами $p, \rho, \mathbf{V}$. Тогда параметры $p ; m^{-2} \cdot \rho ; m \cdot \mathbf{V}$, где функция $m$ должна быть постоянной вдоль каждой линии тока, также будут удовлетворять уравнениям Эйлера (преобразование Прима [16]). Если выбрать какую-нибудь линию тока $\gamma$, то функцию $m$ можно задать так, чтобы она была гладкой по обе стороны от линии $\gamma$, непрерывной на самой линии $\gamma$ и имела бы разрыв нормальной к этой линии производной. Тогда линия $\gamma$ будет линией слабого тангенциального разрыва параметров $p ; m^{-2} \cdot \rho ; m \cdot \mathbf{V}$.

В рассмотренном случае (слабые тангенциальные разрывы) из условия $V_{y}=0$ и из (4) вытекает, что тангенциальная составляющая скорости отлична от нуля. Поэтому, гладкий участок этой линии лежит на линии тока.

Рассмотрев как линии разрыва плотности, так и линии, на которых плотность непрерывна, приходим к следующему выводу. В стационарных дозвуковых течениях газа без точек торможения возможны только тангенциальные разрывы (нормальная к линии разрыва составляющая скорости равна нулю). При этом гладкие участки линий разрывов представляют собой предельные положения линии тока (для слабых разрывов - сами линии тока).

\section{6. Излом линии разрыва}

Продолжим изучение линий разрыва в плоских дозвуковых течениях без точек торможения. Выше были рассмотрены гладкие участки линии разрыва. Но линии разрыва могут быть кусочно-гладкими и иметь точки излома. Оказалось, что в плоских дозвуковых течениях без точек торможения невозможно существование точек излома. Покажем это.

Пусть кусочно-гладкая линия разрыва $\gamma$ не является гладкой и имеет излом во внутренней точке течения $A$. К точке $A$ можно «подойти» по линии $\gamma$ с разных сторон излома. Возможны два случая.

Первый случай. Предельные положения касательных к линии $\gamma$ при приближении к точке $A$ не совпадают. Тогда с каждой стороны от точки $A$ находится гладкий участок линии $\gamma$, представляющий собой тангенциальный разрыв, на котором нормальные составляющие предельных значений скорости равны нулю. Поэтому несовпадение предельных значений углов наклона возможно только в случае, если и тангенциальные составляющие скорости стремятся к нулю при приближении к точке $A$. Это противоречит условию (4), и рассматриваемый (первый) случай невозможен.

Второй случай. Предельные значения углов наклона при приближении к точке $A$ совпадают, но сама линия разрыва $\gamma$ в этой точке имеет излом типа «точка возврата», который напоминает график функции $y=\sqrt{|x|}$ вблизи начала координат. Тогда совпадение предельных значений углов наклона не противоречит условию (4). Но этому условию будет противоречить закон сохранения массы с учетом непрерывности и неравенства нулю компонент вектора $\rho \mathbf{V}$ внутри той «узкой» части достаточно малой окрестности точки $A$, которая вырезана из этой окрестности линией разрыва. Таким образом, этот (второй) случай также невозможен, и если в течении есть линия разрыва и выполнены условия (4) и (5), то эта линия является гладкой и с обеих сторон совпадает с предельными положениями линий тока. Рассматривая равновесие сил, несложно убедиться, что давление на таких линиях разрыва непрерывно [5]. 
Из невозможности существования изломов и углов на линии разрыва следует, что при соблюдении условий (4) и (5) невозможно также и существование точек, из которых выходят несколько линий разрыва. В частности, на линии разрыва не может быть точки, к которой под углом или по касательной подходит другая линия разрыва.

\section{7. Незамкнутые линии разрыва}

Допустим, что линия (тангенциального) разрыва не замкнута и ее начало $A$ находится во внутренней точке течения. Тогда в точке $A$, как и вдоль всей линии разрыва, выполнено условие (4). Учитывая, что как показано выше, нормальная к линии разрыва скорость равна нулю, в точке $A$ линия тока непрерывного течения разделяется на две предельные линии тока, обтекающие линию разрыва с разных сторон. В отличие от предыдущих разделов, обозначим символом $\gamma$ не линию разрыва, а всю линию тока. При этом линия разрыва будет являться частью линии $\gamma$. Точка $A$ лежит на линии $\gamma$ и разграничивает участок с гладкими параметрами и участок линии $\gamma$, который является линией разрыва. Для определенности будем считать, что жидкая частица сначала движется вдоль $\gamma$ по участку с гладкими параметрами и затем разделяется на две частицы, движущиеся вдоль «разрывного» участка $\gamma$ (случай движения частицы в обратном направлении рассматривается аналогично). У разделенных частиц жидкости будут одинаковы энтропийная функция $p \cdot \rho^{-k}$ и полная энтальпия $h=\frac{k}{k-1} \frac{p}{\rho}+\frac{V^{2}}{2}$, поскольку они одинаковы в начале линии разрыва. Вместе с непрерывностью давления на линии тангенциального разрыва это приводит к заключению, что на линии $\gamma$ не может быть разрывов гидродинамических параметров (не может быть сильных разрывов).

Итак, из предположения о том, что линия разрыва не замкнута и ее начало $A$ находится во внутренней точке течения, следует, что «разрывная» часть линии $\gamma$, находящаяся с одной из сторон от точки $A$, является линией тока и, одновременно, линией слабого тангенциального разрыва; а с другой стороны от точки $A$ - «обычной» линией тока, находящейся в области гладких параметров $p, \rho, \mathbf{V}$. Докажем, что это невозможно. То есть докажем, что на всей линии $\gamma$ параметры $p, \rho, \mathbf{V}$ имеют непрерывные пространственные производные. В силу непрерывности этих параметров на линии разрыва, для этого достаточно показать, что непрерывные продолжения их производных с разных сторон линии разрыва совпадают.

Рассмотрим вспомогательное векторное поле - поле касательного к линиям тока вектора $\mathbf{e}=\mathbf{V} /|\mathbf{V}|$. Его непрерывность в точках $\gamma$ следует из того, что это поле с обеих сторон от линии $\gamma$ допускает непрерывное продолжение на линию $\gamma$ и при этом каждое из двух непрерывных продолжений совпадает с касательным вектором к линии $\gamma$. Гладкость поля е вне линии $\gamma$ следует из гладкости скорости. Покажем, что и на линии $\gamma$ поле вектора $\mathbf{e}=\mathbf{V} /|\mathbf{V}|$ является гладким (непрерывно дифференцируемым). Расположим начало прямоугольной декартовой системы координат $O x y$ в произвольной точке линии $\gamma$. В частности, точка $O$ может совпадать с точкой $A$. Ось $O x$ направим по касательной к этой линии. В силу расположения осей системы $O x y$, в начале координат $O$ непрерывны производные $p, \rho, \mathbf{V}$ по координате $x$. Рассуждая как при выводе уравнений (6) и учитывая, что в нача- 
ле координат $V_{y}=0$, приходим к выводу, что в начале координат $O$ непрерывны следующие производные $\frac{\partial}{\partial y} p$ и $\frac{\partial}{\partial y} V_{y}$.

Итак, в точке $O$ непрерывны сами гидродинамические параметры ( $p, \rho, \mathbf{V})$, их производные по координате $x$ (в частности $\frac{\partial}{\partial x} V_{x}$ и $\frac{\partial}{\partial x} V_{y}$ ) и две производные по координате $y$ (а именно, $\frac{\partial}{\partial y} p$ и $\left.\frac{\partial}{\partial y} V_{y}\right)$. Осталось показать, что непрерывны $\frac{\partial}{\partial y} V_{x}$ и $\frac{\partial}{\partial y} \rho$.

Поскольку $e_{y}=V_{y} / \sqrt{V_{x}^{2}+V_{y}^{2}}$, имеем

$$
\frac{\partial}{\partial y} e_{y}=\left(V_{x}^{2}+V_{y}^{2}\right)^{-\frac{1}{2}} \frac{\partial}{\partial y} V_{y}-\left(V_{x}^{2}+V_{y}^{2}\right)^{-\frac{3}{2}}\left(V_{y}^{2} \frac{\partial}{\partial y} V_{y}+V_{y} V_{x} \frac{\partial}{\partial y} V_{x}\right)
$$

В правой части этого равенства в точке $O$ непрерывны все величины, кроме, может быть, $\frac{\partial}{\partial y} V_{x}$. Однако эта производная в выражении (7) умножена на величину $V_{y}$, которая стремится к нулю при приближении к точке $O$. Учитывая ограниченность предельного значения $\frac{\partial}{\partial y} V_{x}$, получаем, что оба предельных значения $\frac{\partial}{\partial y} e_{y}$ в точке $O$ одинаковы и конечны (речь идет о пределах с разных сторон линии $\gamma$ ). Поэтому производная $\frac{\partial}{\partial y} e_{x}$ непрерывна в точке $O$.

Вне линии $\gamma$ компоненты $e_{x}$ и $e_{y}$ непрерывно дифференцируемы и верно тождество $e_{x}^{2}+e_{y}^{2}=1$. Из этого тождества следует дифференциальное равенство: $e_{x} \frac{\partial}{\partial y} e_{x}+e_{y} \frac{\partial}{\partial y} e_{y}=0$. При приближении к точке $O$ компонента $e_{x}$ стремится к единице, а компонента $e_{y}-$ к нулю. Поэтому из конечности предела $\frac{\partial}{\partial y} e_{y}$ и из упомянутого дифференциального равенства следует, что $\frac{\partial}{\partial y} e_{x}$ стремится к нулю при приближении к точке $O$ по любой линии, не пересекающей линию $\gamma$. Следовательно, производная $\frac{\partial}{\partial y} e_{x}$ непрерывна в точке $O$.

Далее, поскольку $e_{x}=V_{x} / \sqrt{V_{x}^{2}+V_{y}^{2}}$, имеем

$$
\frac{\partial}{\partial y} e_{x}=\left(V_{x}^{2}+V_{y}^{2}\right)^{-\frac{1}{2}} \frac{\partial}{\partial y} V_{x}-\left(V_{x}^{2}+V_{y}^{2}\right)^{-\frac{3}{2}}\left(V_{x} V_{y} \frac{\partial}{\partial y} V_{y}+V_{x}^{2} \frac{\partial}{\partial y} V_{x}\right)
$$


С учетом конечности предельного значения $\frac{\partial}{\partial y} V_{x}$, равенства $V_{y}=0$ и только что доказанной непрерывности $\frac{\partial}{\partial y} e_{x}$ из (8) следует, что производная $\frac{\partial}{\partial y} V_{x}$ будет непрерывна в точке $O$. Что касается непрерывности в точке $O$ производных $\frac{\partial}{\partial x} e_{x}$ и $\frac{\partial}{\partial x} e_{y}$, то она следует из непрерывности $\frac{\partial}{\partial x} V_{x}$ и $\frac{\partial}{\partial x} V_{y}$ и из выражений для $\frac{\partial}{\partial x} e_{x}$ и $\frac{\partial}{\partial x} e_{y}$, аналогичных (7) и (8).

Расположение начала координат было выбрано произвольно. Поэтому пространственные производные $e_{x}$ и $e_{y}$ непрерывны во всех точках линии $\gamma$, кроме, может быть, точки $A$. Следовательно, поле касательного к линиям тока вектора $\mathbf{e}=\mathbf{V} /|\mathbf{V}|$ является гладким (непрерывно дифференцируемым).

Теперь покажем, что внутренняя точка $A$ не может разделять линию тока так, что с одной стороны от точки $A$ эта линия является линией слабого тангенциального разрыва; а с другой стороны от точки $A$ «обычной» линией тока, находящейся в области гладких параметров $p, \rho, \mathbf{V}$. Воспользуемся гладкостью поля $\mathbf{e}=\mathbf{V} /|\mathbf{V}|$ в некоторой окрестности точки $A$. Во-первых, гладкость $\mathbf{e}=\mathbf{V} /|\mathbf{V}|$ и тот факт, что $|\mathbf{e}|=1 \neq 0$, позволяет ввести в некоторой окрестности $U(A)$ ортогональную систему естественных координат Asn с началом в точке $A$ так, чтобы равенства вида $n=$ const задавали бы линии тока, а равенства вида $s=$ const задавали бы линии, ортогональные линиям тока. При этом линия $\gamma$ задается равенством $n=0$. Во-вторых, в силу гладкости поля единичного вектора $\mathbf{e}=\mathbf{V} /|\mathbf{V}|$ гладкость величин $p, \rho, \mathbf{V}$ в какой-либо точке равносильна гладкости в этой точке функций $p=p(s, n)$; $\rho=\rho(s, n)$ и компонент вектор-функции $\mathbf{V}=\mathbf{V}(s, n)$, записанных в системе координат Asn.

Для определенности будем считать, что точки линии $\gamma$ при $s<0$ лежат в области гладких параметров $p, \rho, \mathbf{V}$. Тогда, согласно (3), в этой области будет гладкой энтропийная функция $C=p \cdot \rho^{-k}$. В системе координат Asn эта функция зависит только от одной координаты: $C=C(n)$. Поэтому гладкость $C=p \cdot \rho^{-k}$ равносильна непрерывности $\frac{d}{d n} C(n)$ в окрестности точки $n=0$. Энтропийная функция постоянна вдоль линий тока. Поэтому вид функции $C=C(n)$ одинаков при всех значениях координаты $s$ в рассматриваемой окрестности точки $A$. Следовательно, энтропийная функция является гладкой и с другой стороны от точки $A$, т. е. в точках линии $\gamma$ при $s \geq 0$. Из гладкости давления $p$ и из гладкости функции $C=p \cdot \rho^{-k}$ следует гладкость плотности $\rho$.

Чтобы показать, что при $s \geq 0$ линия $\gamma$ не является слабым тангенциальным разрывом, осталось показать гладкость скорости, которая в системе координат имеет компоненты $V_{s} \neq 0$ и $V_{n} \equiv 0$. Гладкость $V_{n} \equiv 0$ очевидна. В силу непрерывности $V_{s}$ на линии $\gamma$, на этой линии будет непрерывна и $\frac{d}{d s} V_{s}$. Доказательство непрерывности $\frac{d}{d n} V_{s}$ можно построить аналогично доказательству гладкости плотности, используя уже доказанный факт гладкости 
$\rho$ и свойство, следующее из (1) и (3), состоящее в том, что полная энтальпия $h=\frac{k}{k-1} \frac{p}{\rho}+\frac{V_{s}^{2}}{2}$ зависит только от одной координаты: $h=h(n)$. Приводить это доказательство представляется излишним.

Таким образом, в плоских дозвуковых течениях без точек торможения незамкнутые линии сильных и слабых разрывов не могут заканчиваться во внутренних точках течения и, если область течения ограничена, обязательно достигают его границы.

\section{8. Замкнутые линии разрыва}

Рассмотрим замкнутые линии разрывов. Они ограничивают подобласти с гладкими параметрами. Внутри подобласти течения с гладкими параметрами может содержаться «вырез» - другая подобласть с аналогичными свойствами. В «вырезе» может содержаться следующий «вырез» и так далее. Но поскольку количество таких «вырезов» конечно, существует односвязная область $G_{1}$, не содержащая «вырезанной» подобласти. Введем прямоугольную декартову систему координат $O x y$ с началом внутри области $G_{1}$. Зададим в замыкании области $G_{1}$ функцию

$$
\psi\left(x_{1}, y_{1}\right)=\int_{(0,0)}^{\left(x_{1}, y_{1}\right)} \rho V_{y} d x-\rho V_{x} d y,
$$

где интегрирование ведется по произвольной кривой, расположенной внутри $G_{1}$ и соединяющей точку $\left(x_{1}, y_{1}\right)$ с началом координат $(0,0)$. Такое задание функции $\psi$ корректно, поскольку область $G_{1}$ односвязна, а интеграл, в силу уравнения неразрывности и формулы Грина, не зависит от пути интегрирования.

На границе $\partial G_{1}$ (непрерывное) продолжение скорости ни в одной точке не обращается в ноль, так как иначе было бы нарушено условие (4). Это непрерывное продолжение скорости является касательным вектором к границе $\partial G_{1}$. По построению функции $\psi$, ее градиент перпендикулярен вектору $\mathbf{V}$. Поэтому градиент $\psi$ перпендикулярен границе $\partial G_{1}$ во всех ее точках. То есть функция $\psi$ имеет постоянное значение на $\partial G_{1}$. Это значит, что в $G_{1}$ существует внутренняя точка, в которой обе пространственные производные функции $\psi$ равны нулю. Учтем, что производные этой функции с точностью до знака являются компонентами вектора $\rho \mathbf{V}$. Согласно «гипотезе», плотность во внутренней точке подобласти с гладкими параметрами не может обращаться в ноль. Следовательно, во внутренней точке области $G_{1}$ есть точка нулевой скорости. Это противоречит условию (4).

Полученное противоречие означает невозможность существования в плоских дозвуковых течениях без точек торможения замкнутой линии разрыва, расположенной внутри односвязной области течения.

\section{9. Достаточное условие невозможности существования разрывов при течении газа в канале}

Выше показано, что в плоских дозвуковых течениях без точек торможения, происходящих в ограниченных односвязных областях, линии разрывов обязательно достигают границы. Если рассмотреть течения в плоских каналах с гладкими стенками, то часть границы, 
совпадающая со стенками, является линией тока. Рассуждения, аналогичные тем, которые были проведены в разделе 4 (о невозможности изломов), приводят к заключению, что линии разрыва не могут достигать стенок канала.

Таким образом, достаточным условием отсутствия разрывов при дозвуковом течении без точек торможения в плоском односвязном канале является отсутствие сильных и слабых разрывов во входном и выходном сечениях.

Полезность этого вывода состоит, в частности, в правомочности применения к такому течению дозвукового принципа максимума скорости [2], признака наличия точек торможения [17] и принципа максимума давления [18]. Эти три свойства верны при условии непрерывности вторых производных, что обеспечивается, как показано выше, отсутствием разрывов.

\section{0. Заключение}

Проведен анализ полных уравнений Эйлера плоского стационарного течения идеального совершенного газа, которое может быть вихревым, а энтропия может быть различной на различных линиях тока. Кроме того, в течении допускается существование линий сильных и слабых разрывов. Обоснована содержательность рассмотрения таких течений в постановке, которая названа гипотезой непрерывной сплошной среды для плоских стационарных течений идеального совершенного газа. На основе положений этой гипотезы показано, что достаточным условием отсутствия разрывов при дозвуковом течении без точек торможения в плоском канале является отсутствие сильных и слабых разрывов во входном и выходном сечениях.

\section{Благодарность}

Автор выражает проф. А.Л. Стасенко (ЦАГИ) и доченту Г.Б. Сизых (МФТИ) за обсуждение гипотезы и полезные замечания.

\section{Литература}

1. Лойцянский Л. Г. Механика жидкости и газа. - М. : Дрофа, 2003. - 840 с.

2. Берс Л. Математические вопросы дозвуковой и околозвуковой аэродинамики. - М. : Изд-во иностр. лит., 1961. - 208 с.

3. Кочин Н. Е., Кибель И. А., Розе Н. В. Теоретическая гидромеханика. Ч. 1. - М. : Физматгиз, 1963. - 584 с.

4. Бэтчелор Дж. Введение в динамику жидкости. - М. : Мир, 1973. - 760 с.

5. Седов Л. И. Механика сплошной среды : в 2-х т. - М. : Наука, 1983.

6. Никольский А. А., Таганов Г. И. Движение газа в местной сверхзвуковой зоне и некоторые условия разрушения потенциального течения // ПММ. - 1946. - Т. 10, вып. 4. C. 481-502.

7. Hopf E. Elementare Bemerkungen über die Lösungen partieller Differentialgleichungen zweiter Ordnung vom Elliptischen Typus // Sitzungsberichte der Preussischen Akademie der Wissenschaften. - 1927. - Vol. 19. - P. 147-152.

8. Миранда К. Уравнения с частными производными эллиптического типа. - М. : Изд-во иностр. лит., 1957. - 256 с.

9. Gilbarg D., Shiffman M. On bodies achieving extreme value of the critical Mach number. I // J. Rat. Mech. and Analysis. - 1954. - Vol. 3, no. 2. - P. 209-230.

10. Kraiko A. N. Planar and axially symmetric configurations which are circumvented with the maximum critical mach number // Journal of Applied Mathematics and Mechanics. - 1987. Vol. 51, iss. 6. - P. 723-730. - DOI: 10.1016/0021-8928(87)90131-6. 
11. Rosenhead L. The formation of vortices from a surface of discontinuity // P. Roy. Soc. Lond. - 1931. - A134. - P. 170-192.

12. Белоцерковский С. М., Ништ М. И. Отрывное и безотрывное обтекание тонких крыльев идеальной жидкостью. - М. : Наука, 1978. - 352 с.

13. Cottet G.-H., Koumoutsakos P. Vortex methods: theory and practice. - Cambridge University Press, 2000. - 320 p.

14. Gutnikov V. A., Lifanov I. K., Setukha A. V. Simulation of the Aerodynamics of Buildings and Structures by Means of the Closed Vortex Loop Method // Fluid Dynamics. - 2006. - Vol. 41, no. 4. - P. 555-567. - DOI: 10.1007/s10697-006-0073-4.

15. Фихтенгольц Г. М. Курс дифференциального и интегрального исчисления : в 3 т. T. II - М. : Физматлит, 2001. - 864 с.

16. Munk M., Prim R. On the multiplicity of steady gas flows having the same streamline pattern // Proc. Nat. Acad. Sci. USA. - 1947. - Vol. 33. - P. 137-141.

17. Сизых Г. Б. Признак наличия точки торможения в плоском безвихревом течении идеального газа // Труды МФТИ. - 2015. - Т. 7, № 2 (26). - С. 108-112.

18. Голубкин В. Н., Сизых Г. Б. Экстремальные свойства давления в плоских дозвуковых течениях // Труды МФТИ. - 2016. - Т. 8, № 4 (32). - С. 149-154. 